\title{
The impact of hotspot-targeted interventions on malaria transmission: study protocol for a cluster-randomized controlled trial
}

Teun Bousema ${ }^{1,2^{*}+}$, Jennifer Stevenson ${ }^{3 \dagger}$, Amrish Baidjoe ${ }^{2}$, Gillian Stresman ${ }^{1}$, Jamie T Griffin ${ }^{4}$, Immo Kleinschmidt ${ }^{5}$, Edmond J Remarque ${ }^{6}$, John Vulule ${ }^{7}$, Nabie Bayoh $^{7}$, Kayla Laserson $^{7,8}$, Meghna Desai ${ }^{7,8}$, Robert Sauerwein ${ }^{2}$, Chris Drakeley ${ }^{1}$ and Jonathan Cox $^{3}$

\begin{abstract}
Background: Malaria transmission is highly heterogeneous in most settings, resulting in the formation of recognizable malaria hotspots. Targeting these hotspots might represent a highly efficacious way of controlling or eliminating malaria if the hotspots fuel malaria transmission to the wider community.

Methods/design: Hotspots of malaria will be determined based on spatial patterns in age-adjusted prevalence and density of antibodies against malaria antigens apical membrane antigen-1 and merozoite surface protein-1. The community effect of interventions targeted at these hotspots will be determined. The intervention will comprise larviciding, focal screening and treatment of the human population, distribution of long-lasting insecticide-treated nets and indoor residual spraying. The impact of the intervention will be determined inside and up to $500 \mathrm{~m}$ outside the targeted hotspots by PCR-based parasite prevalence in cross-sectional surveys, malaria morbidity by passive case detection in selected facilities and entomological monitoring of larval and adult Anopheles populations.

Discussion: This study aims to provide direct evidence for a community effect of hotspot-targeted interventions. The trial is powered to detect large effects on malaria transmission in the context of ongoing malaria interventions. Follow-up studies will be needed to determine the effect of individual components of the interventions and the cost-effectiveness of a hotspot-targeted approach, where savings made by reducing the number of compounds that need to receive interventions should outweigh the costs of hotspot-detection.
\end{abstract}

Trial registration: NCT01575613. The protocol was registered online on 20 March 2012; the first community was randomized on 26 March 2012.

Keywords: Anopheles, elimination, epidemiology, eradication, falciparum, heterogeneity, immunology, malaria, molecular, transmission

\section{Background}

The transmission of infectious agents is highly heterogeneous in space and time. For many infectious diseases, a small number of human hosts are most frequently or most heavily infected while the majority of a local population is much less affected [1-4]. In malaria, this heterogeneity of disease transmission often results in variation

\footnotetext{
* Correspondence: teun.bousema@lshtm.ac.uk

${ }^{\dagger}$ Equal contributors

'Department of Immunology \& Infection; Faculty of Infectious and Tropical Diseases, London School of Hygiene and Tropical Medicine, London, UK ${ }^{2}$ Radboud University Nijmegen Medical Centre, Nijmegen, the Netherlands Full list of author information is available at the end of the article
}

in malaria incidence within small areas [5-10]. In some settings the non-random distribution of malaria incidence between households appears to conform to the '20/80 rule' [2], whereby approximately $20 \%$ of a host population contributes $80 \%$ of the cases of an infectious organism $[5,9]$. The factors underlying the micro-epidemiology of malaria are not fully understood but include variation in distance from the nearest mosquito breeding site [5-9,11], wind direction[12], house construction features [6,8,9,13,14], human behavioural $[7,8,13]$ and genetic factors $[7,8,15]$.

Heterogeneity in malaria transmission has implications for malaria control. Individuals who are bitten most

\section{Biomed Central}


often are most likely to be infected and can amplify transmission by infecting a large number of mosquitoes with malaria parasites. Estimates of the basic reproductive number $\left(R_{0}\right)$, a central concept in infectious disease epidemiology defined as the average number of secondary cases arising in a susceptible population as a result of a single human case over the course of their infection, are sensitive to assumptions of heterogeneous mosquito exposure. $R_{0}$ may be four times higher when heterogeneous mosquito exposure, as opposed to homogeneous exposure, is considered $[2,4,16]$.

The large influence of heterogeneous exposure on malaria transmission also suggests that interventions targeting areas of comparatively high exposure can be highly effective. Woolhouse and colleagues suggested that, depending on the costs of identifying hotspots of transmission, treating the core $20 \%$ might be preferable to non-targeted interventions on economic grounds [2]. If hotspots fuel transmission to a wider geographical region, community protection may be achieved by targeting those individuals that are most important for disease transmission. This hotspot-targeted approach will be most (cost) effective if the assumption that hotspots fuel transmission in surrounding areas is correct - and then only if such hotspots can be reliably detected [4]. Several approaches to identify hotspots of malaria transmission have been proposed in recent years. Annual incidence of clinical malaria is a frequently used indicator of hotspots of malaria transmission [8-10] but is affected by a differential acquisition of protective immune responses inside and outside hotspots $[17,18]$. Geographical clustering of asymptomatic parasite carriage may be a more stable indicator of hotspots of transmission [10] and in areas of moderate or low endemicity hotspots might be most readily detected using serological markers of malaria exposure [9,10,19-22]. In an area of moderate endemicity in Tanzania, serological data have been used to identify clinically and entomologically confirmed hotspots of malaria transmission with $96 \%$ sensitivity and $82 \%$ specificity [9].

This manuscript describes a methodological approach to identifying hotspots of malaria transmission and a protocol for the evaluation of a hotspot-targeted intervention. The aim of this intervention study is to determine whether the simultaneous roll-out of interventions in hotspots of malaria transmission has a communitywide effect that extends beyond the hotspot boundaries and results in local reduction and possibly elimination of malaria.

\section{Methods/design Study area}

The study will be conducted in highland fringe localities (1400 m to $1600 \mathrm{~m}$ altitude) in Rachuonyo South
District, Western Kenya $\left(34.75\right.$ to $34.95^{\circ} \mathrm{E}, 0.41$ to $\left.0.52^{\circ} \mathrm{S}\right)$. The predominant ethnicity in Rachuonyo is Luo. Local residents depend upon farming, cattle and goat herding for subsistence [23]. Compounds comprise an average of two houses (25th to 75th percentile 1 to 3 ) and are distributed broadly across a rolling landscape intersected with small streams and rivers. The main malaria vectors in the area are Anopheles gambiae s.s., An. arabiensis, and An. funestus. Malaria transmission is seasonal, with two peaks in malaria cases reflecting the bimodal rainfall pattern; a peak corresponding to the heaviest rainfall typically occurs between March and June and there is a smaller peak between October and November each year. Most malaria is caused by Plasmodium falciparum [23]. Community cross-sectional surveys conducted in 2010 indicated parasite prevalence averaging $14.8 \%$ in the general population but varying between localities from $0 \%$ and $51.5 \%$. School surveys carried out in primary schools in the same year indicated an average parasite prevalence of $25.8 \%$ in 7 to 18 year olds (minimum and maximum for individual schools 0 to $71.4 \%$ ). Insecticide-treated nets (ITNs) have been promoted by the Ministry of Public Health and Sanitation for many years and distribution campaigns have taken place through antenatal and child health clinics, reaching net ownership for under $5 \mathrm{~s}$ of $82.7 \%$, as determined in surveys in 2010 (unpublished data). In addition, community-wide mass distribution of ITNs was undertaken by the Division of Malaria Control (DOMC) in 2011. Indoor residual spraying (IRS) with a pyrethroid was first carried out in Rachuonyo South in mid 2008 with financial support of the US President's Malaria Initiative. Reported house coverage with IRS in Rachuonyo South was estimated at 70.3\% in 2009 and $74.3 \%$ in 2010.

\section{Sampling strategy to identify hotspots of transmission}

We will select a $5 \times 20 \mathrm{~km}\left(100 \mathrm{~km}^{2}\right)$ area within which results from recent community and school malaria surveys suggest highly heterogeneous malaria exposure. The study area will be divided into 400 cells of $500 \times 500 \mathrm{~m}$ that will be further subdivided into four sub-cells of $250 \times 250 \mathrm{~m}$.

All structures in the area have been geo-located using contemporaneous high-resolution satellite data (Quickbird; DigitalGlobe Services, Inc., Denver, CO, USA), which were acquired and processed using standard digital image processing techniques (ENVI 4.8, Exelis Visual Information Solutions, McLean, VA, USA). Pansharpened colour images were then imported into a geographic information system (ArcGIS 9.2; Environmental Systems Research Institute, Redlands, CA, USA) and all structures digitized manually, giving a total of 8,632 structures with a median of 45 (25th to 75 th percentile, 35 to 52$)$ per $500 \times 500 \mathrm{~m}$ cell. We aim to obtain measurements from $\geq 50$ individuals per $500 \times 500 \mathrm{~m}$ cell, since estimates of sero-conversion rates from fewer than 
50 observations from all age groups combined are likely to be unreliable [9]. To maximize the discriminative power of serological markers of exposure, we will sample individuals in predefined age strata ( $\leq 5$ years; 6 to 10 years; 11 to 15 years; 16 to 25 years and $>25$ years). For logistical reasons, our unit of sampling will be the compound.

To limit the chances of two selected structures belonging to the same compound, an iterative sampling approach will be used that involves randomly selecting a 'seed' structure and then removing all closely neighbouring structures (within $50 \mathrm{~m}$ ) from the sample universe before proceeding to select a second structure. This process will be repeated until all possible 'non-neighbouring' structures have been selected. From the resulting list of eligible structures a sample of 16 structures will be chosen from each $500 \times 500 \mathrm{~m}$ cell. To ensure maximum geographical coverage, at least one compound will be selected from each $250 \times 250 \mathrm{~m}$ sub-cell, while the number of compounds selected from each of the sub-cells will be weighted by the structure density in these sub-cells.

All other structures in which people sleep and which are associated with each selected compound will be included. The target number of 50 observations per $500 \times 500 \mathrm{~m}$ cell is chosen irrespective of the population density of the cells.

\section{Data collection and measurements to identify hotspots of transmission \\ Enumeration}

For planning purposes, the field area will be subdivided into 20 blocks of $5 \times 4$ cells (that is, a block is $2.5 \times 2$ $\mathrm{km}$ in size). Teams will be provided with a printed overview map of the block they are working in (Figure 1), as well as detailed high-resolution maps incorporating the QuickBird satellite data for each $500 \times 500 \mathrm{~m}$ cell. Each team will also be provided with a handheld global positioning system (GPS) receiver (Garmin 62S; Garmin International, Inc., Olathe, KS, USA) that has been preloaded with the selected compound positions and cell boundaries. An enumeration team, comprising one field worker, a reporter and a local guide, will visit selected compounds to explain the study procedures, enumerate inhabitants, collect information on house characteristics and inform residents that the survey team will visit later that day. In situations where none of the structures within a selected compound corresponds with a residential building, the selected compound will be replaced with the nearest visible inhabited compound. The location of this replacement compound will be recorded on the satellite images, mapped using the GPS and recorded on the enumeration forms.

All compounds where at least one adult ( $>20$ years) and one child $(<15$ years) are permanent residents (defined as sleeping regularly in the structure) qualify for enrolment. For compounds with fewer residents, replacements will be made, as described. If the head of the compound agrees to participate, the geographical coordinates of the main house of the compound will be recorded and compound and individual house codes will be written on the doors of all sleeping structures with a permanent marker. The names and ages of all compound members will be recorded on study forms and information on compound and house characteristics, including structure type, ITN coverage, and IRS history, will be collected using a precoded questionnaire (Programmed in Visual Basic, Visual CE v11.0) on a personal digital assistant (HP Ipaq 210, Windows Mobile 6.1). A personal study identification (ID) card will be issued to each individual, which has to be shown to the sampling team when they visit later that same day.

The field workers will carry a checklist to record the cumulative number of selected individuals for each age category. The order in which compounds are visited will be randomly selected based on a computer-generated list. After completing a compound, the enumeration team continues to the next compound until at least ten compounds have been enumerated. If the checklist indicates that age targets are not met at this point, they will continue visiting compounds according to the list until each age target is met.

\section{Sampling}

After enumeration, participating compounds will be visited by a sampling team consisting of two fieldworkers trained in consenting, interviewing and sampling techniques. Sampling teams will be provided with relevant maps, compound lists, enumeration forms and ID cards in advance. Compounds will be located using the names of the compound heads and by codes marked on doors at the point of enumeration; compound occupants will be asked to present their ID card for formal confirmation. Informed consenting will be conducted and the name, sex, age, residency and travel history, ITN use and sleeping times of each compound member will be recorded. The axillary temperature of each compound member will be measured by digital thermometer (Etos, Zaandam, the Netherlands). For all febrile individuals $\left(>37.2^{\circ} \mathrm{C}\right.$ axillary temperature), a rapid diagnostic test (RDT; Paracheck ${ }^{\circledR}$, Orchid Biomedical Systems, Goa, India) to detect $P$. falciparum-specific histidine rich protein-2 will be performed. For all individuals surveyed, a single finger prick sample will be taken for haemoglobin $(\mathrm{Hb})$ measurement using a HemoCue photometer (HemoCue 201+, Angelholm, Sweden) and three droplets of blood transferred onto a filter paper (3MM Whatman, Maidstone, UK) for serum and DNA collection. After transfer to a field laboratory, filter papers will be dried overnight and stored in plastic bags with silica 


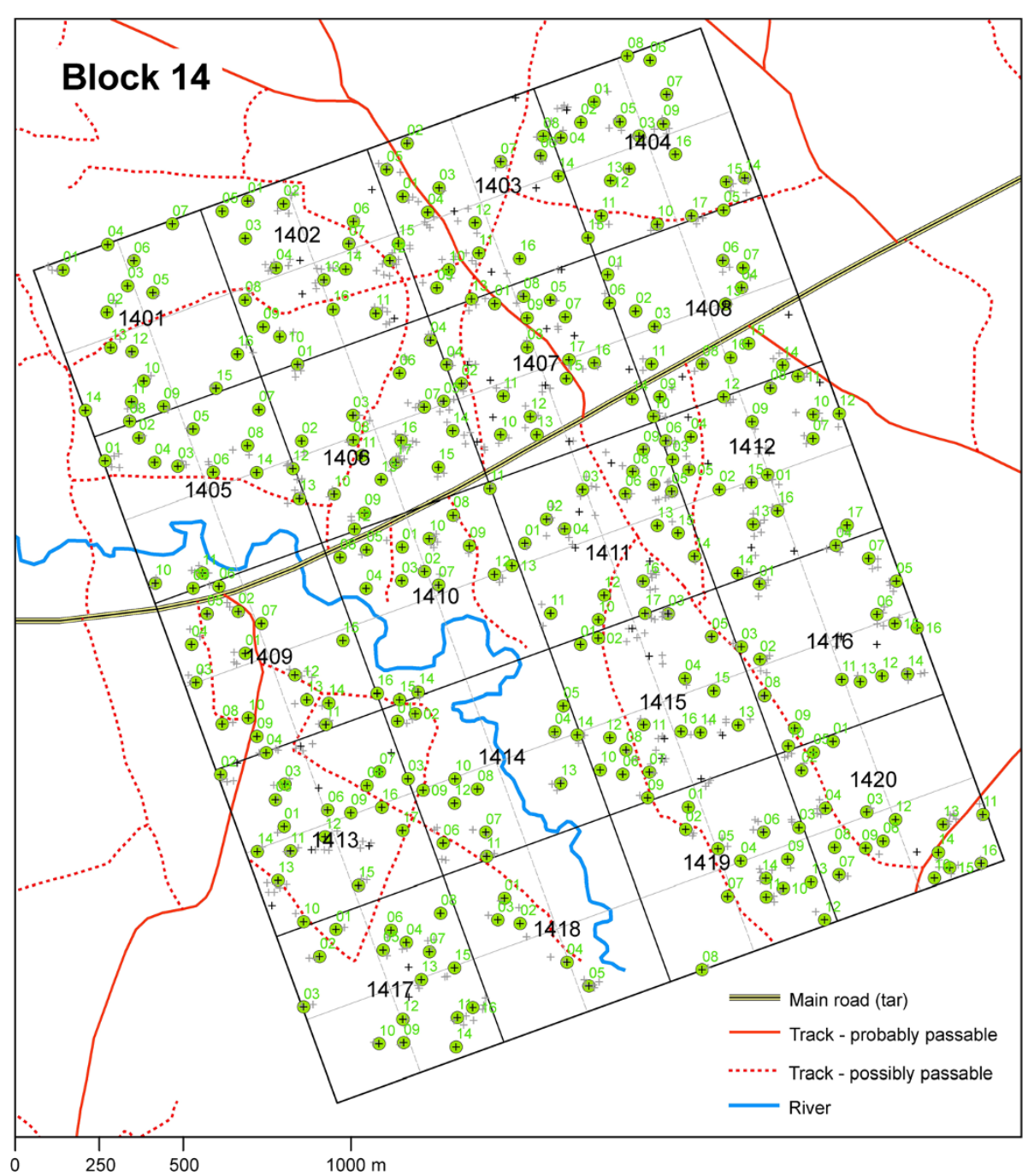

Figure 1 Overview map of one block in the study area comprising 20 cells. A map of a $2 \times 2.5 \mathrm{~km}$ section of the study area that comprises $20500 \times 500$ m cells and 80 sub-cells. Cell numbers are given in black bold letters; grey crosses indicate structures; green circles with black crosses indicate selected and numbered households. Rivers and roads are indicated in the map as given in the legend.

gel. Once a week, samples will be transported to the Kenya Medical Research Institute (KEMRI) CDC laboratory in Kisumu and stored at $-20^{\circ} \mathrm{C}$ until further processing. All individuals with an $\mathrm{Hb} \leq 11 \mathrm{~g} / \mathrm{dl}$ will be given hematinics; individuals with an $\mathrm{Hb} \leq 6 \mathrm{~g} / \mathrm{dl}$ will be accompanied to a nearby health centre for further care. Febrile individuals who are found to be parasitaemic by RDT will be given artemether-lumefantrine (AL, Coartem $^{\circledR}$, Novartis, Switzerland); women of childbearing age who are RDT positive will be assessed for pregnancy and offered a pregnancy test if deemed appropriate. Febrile children below 6 months of age and women who are suspected or found to be pregnant or are unwilling to be tested will be transported to the nearest health facility for a full assessment and treatment.

\section{Malaria parasite prevalence}

A combined extraction of DNA and elution of antibodies will be performed on the samples collected. Two discs with a diameter of $2.5 \mathrm{~mm}$ will be cut from the centre of a single filter paper bloodspot using a holepuncher and will be eluted in deep well plates with addition of $1120 \mu \mathrm{l}$ of a $0.5 \%$ saponin/phosphate buffered saline solution (Sigma Aldrich, Gillingham, UK). DNA will be extracted using the protocol described by Plowe et al. [24]; parasites will be detected by nested PCR $[25,26]$.

\section{Serological markers of malaria exposure}

Total immunoglobulin G (IgG) antibodies against $P$. falciparum apical membrane antigen (AMA-1) and merozoite surface protein $1\left(\mathrm{MSP}-1_{19}\right)$ will be detected by ELISA 
using standard methodology [27,28]. Three serological outcome measures will be used to determine spatial patterns in malaria exposure: (i) the combined antibody prevalence, that is, seropositivity for AMA-1 and MSP- $1_{19}$ or for either of the antigens alone; (ii) the age-adjusted $\log _{10}$-transformed optical density (OD) [21,29]; (iii) the age-dependent sero-conversion rate (SCR) for combined AMA-1, MSP-1 19 antibody prevalence [21,27].

\section{Definition of hotspots}

SaTScan software [30] will be used for the detection of spatial clustering in antibody prevalence (Bernouilli model) and $\log _{10}$-transformed age-adjusted OD values (normal probability model). Circular and elliptic windows [30,31] will be used to systematically scan the study area as a whole and segments of the study area using a $2 \times 4 \mathrm{~km}$ rolling window. Hotspots will be allowed to be $<1 \mathrm{~km}$ in radius and include $<25 \%$ of the population of each window scanned. Segments of the study area will be scanned to improve the sensitivity of the scan to detect local hotspots. Local hotspots may not be detected when scanning the area as a whole, since altitude differences in the study area result in variations in average levels of transmission intensity. A hotspot will be defined as an area for which there is strong evidence $(P<0.05)$ that the observed prevalence or density of combined AMA-1 and MSP-1 19 antimalarial antibodies is higher than expected values. Expected values are based on average values for the area as a whole and for the $2 \times 4 \mathrm{~km}$ rolling window.

Since malaria antibodies are relatively long-lived and may indicate current as well as past malaria exposure, parasite prevalence inside and outside hotspots of malaria exposure will be determined by PCR to confirm ongoing transmission in serologically defined hotspots.

\section{Selection of hotspots and evaluation areas}

Since habitation in the study area is fairly evenly distributed, with every $500 \times 500 \mathrm{~m}$ cell having six or more residential structures, clusters are unlikely to be isolated geographically. To minimize the influence of neighbouring hotspots on malaria transmission in selected intervention or control hotspots, we will select hotspots for which there are no other hotspots detected within $1 \mathrm{~km}$ in any direction from the hotspot boundary. The hotspottargeted intervention will be evaluated in the area surrounding the hotspot (evaluation zones). The evaluation zone will comprise the area surrounding the hotspot up to $500 \mathrm{~m}$ from the hotspot boundary in each direction.

\section{Design of the intervention Intervention clusters}

Four interventions will be rolled out in the period preceding the long rainy season: larviciding, focal screening and treatment (FSAT), long-lasting insecticide-treated nets (LLIN) distribution and IRS. The details of interventions, and their timing, have been agreed upon in collaboration with the DOMC of the Kenyan Ministry of Public Health and Sanitation (MOPHS). Ten per cent of households will be visited 1 to 2 weeks after the intervention to assess any short-term side effects of the FSAT, LLINs and IRS. This sampling strategy was not based on sample size calculations but on logistical feasibility; few side effects were expected.

\section{Larviciding}

All permanent aquatic mosquito habitats in intervention hotspots will be mapped using handheld GPS receivers during the dry season. In the period preceding the long rainy season (April), and throughout the long rainy season (until September) all stagnant water bodies (permanent and temporary) inside these hotspots will be treated on a weekly basis with water-dispersible granule formulations of the commercial strains of Bacillus thuringiensis var. israelensis (Bti), VectoBac ${ }^{\circledR}$, which will be provided by Valent BioSciences Corp., Libertyville, IL. Larviciding will be carried out using previously published protocols [32]; the entire hotspot area will be examined for water bodies on a weekly basis, all of which will be included in the intervention. Spot-checks for surviving Anopheline larvae and pupae will be made on a weekly basis.

\section{Focal screen and treatment (FSAT)}

All compounds in hotspots will be visited and the temperature of each individual will be determined. All individuals aged 6 months to 15 years regardless of temperature and all older individuals who are febrile (tympanic temperature $\geq 37.5^{\circ} \mathrm{C}$ ) will be tested for malaria parasites using HRP-2 and pLDH based RDT (First Response $^{\circledR}$, Premier Medical Corporation Ltd., Kachigam, India). If one or more individuals are found to be RDT positive the entire compound will receive a curative dose of AL with the exception of pregnant women and children below 6 months of age. Because of the different times at which treatment is initiated, one treatment dose for three consecutive days will be supervised by the field worker (day 1 ) or community health workers (days 2 and 3). Each observed dose will be given with fatty food ( $>1.5 \mathrm{~g}$ fat) to facilitate absorption. The second daily dose will be taken without direct supervision but advice on taking the treatment with food will be given. Information on any immediate side effects of the AL will be recorded by the community health workers at each visit; all empty blister packs will be collected by community health workers after treatment has been completed to monitor adherence. 


\section{Long-lasting insecticide-treated nets}

All compounds in hotspots will receive one LLIN per two house members according to MOPHS guidelines. LLINs (Permanet ${ }^{\circledR}$ 3.0) were donated by Vestergaard Frandsen (Hanoi, Vietnam). House members will be given leaflets on proper use and maintenance of nets and study personnel will assist in hanging and demonstrate correct use of the LLINs within houses. Usage and retention of study nets will be assessed by questionnaire six weeks after distribution and any missing or badly torn nets will be replaced within two months after distribution.

\section{Indoor residual spraying}

Routine annual IRS with lambda cyhalothrin (ICON) will be undertaken in all structures where people are sleeping. The IRS campaigns are jointly funded by the Government of Kenya and the US President's Malaria Initiative, and implemented by the Research Triangle Institute (RTI) with the MOPHS, DOMC and District Health Management Teams. For this study IRS will follow MOPHS protocols with more intense mobilization, repeated visits and implementation prior to the start of the malaria transmission season (March to April) in intervention hotspots.

\section{Control clusters}

Control clusters will receive the routine malaria control measures, which for 2012 will be the annual IRS programme as detailed and continued case management at health facilities. The IRS is scheduled to take place in April to May 2012. No LLIN distribution campaigns are planned for 2012.

\section{Design of the randomized evaluation Sensitization and recruitment}

Prior to the implementation of the interventions, meetings with district administrative and health representatives in the selected areas will be organized. Community meetings will be held with local chiefs, community elders and opinion leaders, school representatives and church leaders. All compounds in the selected intervention areas will be visited prior to the intervention; the procedures of the interventions and evaluation procedures will be explained to all compound members present. Identification cards will be distributed that will be used for identification purposes during compound visits and for identification of compound members who visit health facilities in the area.

\section{Randomization}

Hotspots with their surrounding evaluation areas will be randomly assigned to the intervention or control arm. This will be done by simple randomization; no stratification by parasite prevalence or altitude will be undertaken. Clusters will be ordered according to their geographical location, from northwest to southeast. Clusters will be entered in Microsoft Excel 2010 in this geographical order; the same programme will be used to generate random numbers for each of the clusters. Fifty percent of the clusters with the lowest random numbers will be assigned to the intervention arm; $50 \%$ with the highest random numbers to the control arm.

\section{Hypotheses and outcomes \\ Hypotheses}

1. Hotspot-targeted interventions combining larviciding, LLINs, IRS and FSAT will reduce malaria transmission inside and outside hotspots of malaria transmission.

2. The community effect of hotspot-targeted interventions, defined as the impact on parasite prevalence in the evaluation zone surrounding the hotspot, is a function of distance from the hotspot boundary.

\section{Primary and secondary outcome measures}

The primary outcome measure is parasite prevalence by PCR in the evaluation zone surrounding malaria hotspots in intervention and control clusters.

Secondary outcome measures are:

1. Parasite prevalence by PCR in the evaluation zone surrounding malaria hotspots in relation to distance to the boundary of hotspots in intervention and control clusters.

2. Indoor and outdoor Anopheles mosquito densities inside and outside hotspots of malaria transmission in intervention and control clusters.

3. The presence of Anopheles larvae in mosquito breeding sites in malaria hotspots in intervention and control clusters.

4. The number of malaria cases reporting at health facilities, coming from intervention and control clusters.

5. Reported side effects and coverage of FSAT, LLINs and IRS.

\section{Evaluation}

\section{Cross-sectional surveys}

Three cross-sectional surveys will be conducted: at baseline prior to the interventions, during the peak transmission season, and at the end of the peak transmission season. For each cross-sectional survey, 25 compounds that are located inside hotspots, 25 compounds that are located $<250 \mathrm{~m}$ from the hotspot boundary and 25 
compounds that are located 250 to $500 \mathrm{~m}$ from the hotspot boundary will be randomly selected. This strategy is expected to give $\geq 100$ individual observations from each of these three areas. To minimize confounding by neighbouring hotspots, an exclusion buffer will be incorporated in the selection of compounds, ensuring a minimum distance of $\geq 500 \mathrm{~m}$ from neighbouring hotspots.

Study teams will visit selected compounds and, subject to obtaining informed consent, collect information from inhabitants of all houses that belong to that compound using personal digital assistants (PDAs). For individuals older than 6 months, tympanic temperature will be measured and a finger prick blood sample $(\sim 300 \mu \mathrm{l})$ will be collected for assessment of haemoglobin concentration using Copack colour scales (COPACK GmbH, Oststeinbek, Germany) and for collection of nucleic acids and serum on Whatman 3MM filter paper (Maidstone, UK). Whole blood will be collected in BD K2EDTA microcontainers (BD Becton, Dickinson and Company, Oxford, UK) in selected clusters for more detailed molecular analyses. A RDT will be used to determine malaria infection for all febrile individuals. Those with a positive RDT will receive $\mathrm{AL}$ or will be referred to a health centre for further care.

\section{Passive case detection}

A passive case detection system will be introduced in government and mission health facilities to monitor individuals presenting with malaria. Facilities will be selected to cover intervention and control clusters. For this, the catchment areas of health facilities in the area have been determined. Individuals from intervention and control clusters will be asked to present a household card whenever visiting a health facility. This household card will be linked to geo-located compounds. For individuals who present without a household card, other information that allows geo-location will be collected, such as nearest school. Tympanic temperature will be measured, and an RDT used to determine parasite carriage for each individual with measured or reported fever.

\section{Entomological monitoring}

In a subset of the control and intervention clusters, larval and adult mosquito abundance will be monitored. Within each hotspot, a random selection of 15 water bodies along a randomly selected transect will be mapped and the presence or absence of early and late stage Anopheline larvae and pupae will be assessed using a $250 \mathrm{ml}$ mosquito dipper. Five dips will be made in sites smaller than $5 \mathrm{~m}^{2}$; ten dips in sites larger than $5 \mathrm{~m}^{2}$. This will be carried out at two-weekly intervals. Adult collections of Anopheline will be carried out at the same time in 36 randomly selected houses in each cluster selected in cross-sectional surveys. Twelve of these houses will be selected within the hotspots, of which four will be sampled by pyrethrum spray catch (PSC), four for indoor light-trap collections and four for outdoor light-trap collections. Outside the hotspot 24 houses will be randomly selected of which eight will be sampled by PSC, eight for indoor and eight for outdoor light traps.

Pyrethrum spray catching will be carried out indoors according to standard WHO protocols [33]. CDC miniature light traps (Model 512; John W. Hock Company, Gainesville, FL, USA) will be used following previously published procedures to sample mosquitoes indoors [34] and outdoors [35]. The effective range of CDC light traps for outdoor mosquito sampling has been estimated as $5 \mathrm{~m}$ [36]. Accordingly, outdoor sampling will take place $20 \mathrm{~m}$ from selected houses to prevent inhabitants acting as unshielded bait. All traps will be set at 1830 hours and collected at 0630 hours. A collection bottle rotator (Model 1512, John W. Hock Company, Gainesville, FL, US) will be fitted to eight randomly selected light traps set indoors and outdoors within and outside the hotspot; this allows collection cups to rotate every two hours to estimate vector abundance at intervals throughout the night. Vector abundance, parity rates and the proportion of Anopheline females unfed, fed, gravid, and infected will be determined for each species [37] and compared between the two study arms.

\section{Statistical considerations Sample size}

All available malaria simulation models indicate that malaria transmission in the area surrounding intervention hotspots will decrease considerably because malaria transmission is effectively interrupted in those compounds that seed transmission to a larger geographical area $[2,16,38]$. However, there are no published studies that quantify the impact of hotspot-targeted interventions. We estimated the predicted impact of targeted interventions in our study area using one of the leading individual-based simulation models [38], using human, entomological and parasitological characteristics collected at our sites in Kenya. We modelled three scenarios in situations with a pre-intervention parasite prevalence in the human population of 10 to 20\%: (i) no additional interventions; (ii) targeted distribution of LLINs, reaching $90 \%$ of the population in hotspots and (iii) targeted LLINs and targeted effective IRS reaching $90 \%$ of the population in hotspots (Figure 2). The impact of larviciding is currently insufficiently described to be included in the model [38].

Our simulations show that targeted interventions can interrupt transmission completely, both inside and outside hotspots of malaria transmission, reducing overall parasite prevalence to $<5 \%$, in a manner that appears sustainable in the following years (see Figure 2). These predictions have to be interpreted with caution, since (i) 


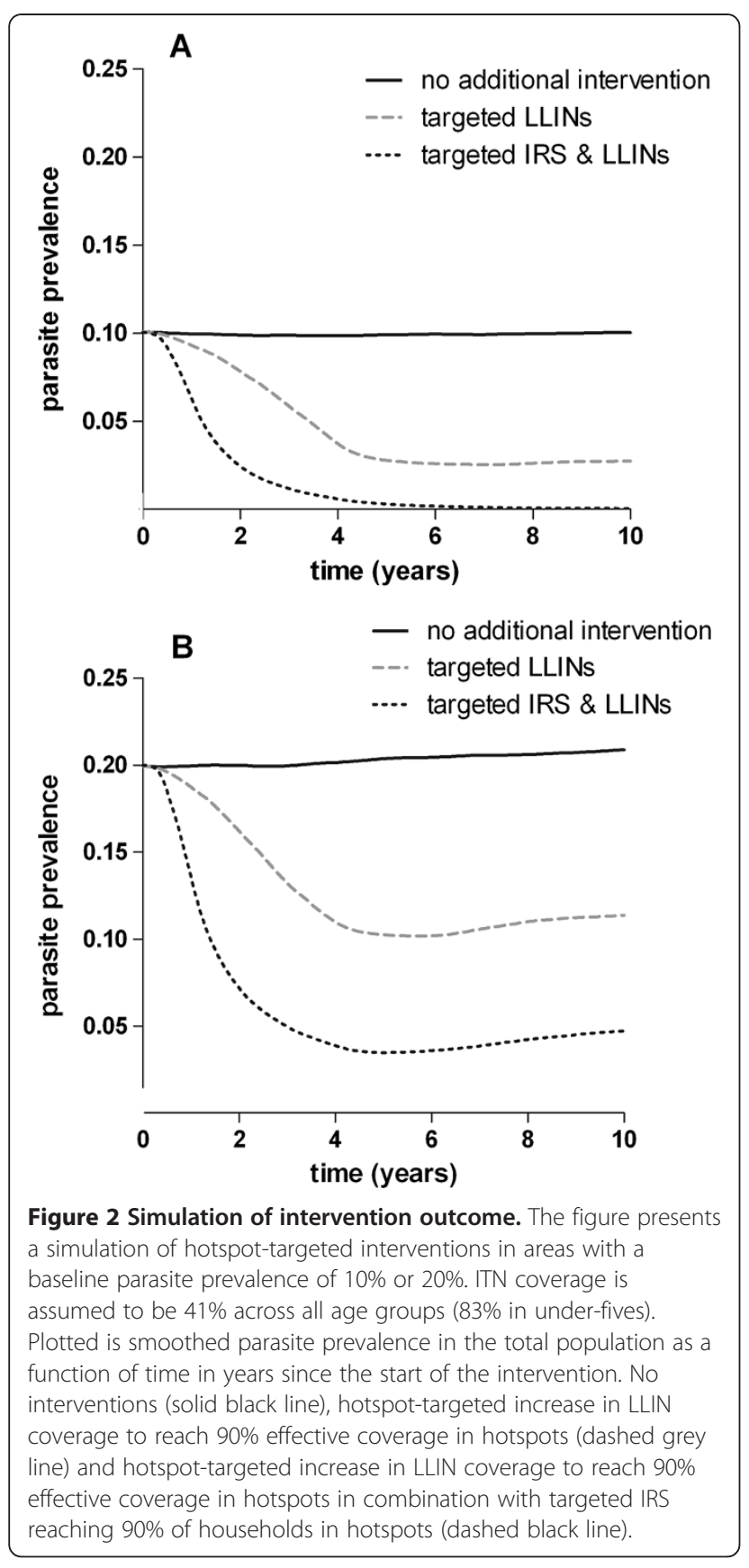

the simulation model has not been prospectively tested; (ii) there is no published evidence that quantifies the impact of hotspot-targeted interventions on transmission intensity in the wider community; and (iii) the intensity of transmission will be highly variable between hotspots in our study area. There is insufficient evidence on which to base power calculations for a cluster-randomized trial; however, these simulations can give an indication of the size of the effect of the planned interventions. The primary outcome measure is parasite prevalence in the evaluation zone. A previous study on the community benefits of ITNs in Asembo, Western Kenya, indicated that an indirect beneficial effect on malaria transmission is most pronounced within $500 \mathrm{~m}$ of the intervention area [39]. We used this finding to define our evaluation zone surrounding the hotspots. Assuming a sample of 200 randomly selected individuals in the evaluation zone of each cluster, a coefficient of variation of true proportions between clusters within each group $(k)$ of 0.4 and mean parasite prevalence of $15 \%$ and $\leq 5 \%$ in the control and intervention clusters respectively, would require five clusters per study arm for $80 \%$ power and $5 \%$ significance level [40]. This power calculation is based on a comparison between arms and the assumption that parasite prevalence will remain unaltered in the control arm.

To estimate the impact of the interventions on the hotspots themselves a sample size of 100 individuals in each of five clusters (hotspots) per study arm, will be required to detect a similar difference between intervention and control clusters ( $\leq 5 \%$ versus $15 \%$ mean prevalence) , assuming $k=0.5,80 \%$ power and $5 \%$ significance.

The entomologic sampling approach was based on previous data from PSC studies carried out by the project where the mean number of female Anophelines caught per house was 0.36 . To detect an $80 \%$ reduction in mean number of female Anophelines caught by PSC in intervention houses compared with control houses at $80 \%$ power and a significance level of 5\%, 213 houses would have to be sampled in each arm. Data from lighttrap studies carried out to date have shown a mean of 1.12 female Anophelines caught indoors per trap per night and so to detect an $80 \%$ reduction in mean number of female Anophelines compared with control houses at $80 \%$ power and a $5 \%$ significance level, 81 traps would have to be set in each arm per month.

\section{Data analysis}

Statistical analyses will be performed in Stata version 12 (StataCorp LP, College Station, TX, USA). The primary analysis will be based on intention to treat whereby all evaluation areas are included in the analysis, regardless of the level of coverage. The main outcome measure, parasite prevalence, will be analyzed as binary variable. For the primary study outcome, we will compare parasite prevalence in the evaluation zones of intervention and control clusters using multilevel mixed-effects logistic regression to account for clustering per compound and random effects to account for differences between study clusters [41]. For secondary study outcomes, we will relate parasite prevalence to distance to the hotspot boundary in meters and in bins of $100 \mathrm{~m}$; this analysis will be done for each of the clusters separately by generalized estimating equations (GEE), adjusting for correlations between observations from the same compound. Indoor and outdoor Anopheles densities will be compared between 
study arms using GEE models and Poisson or negative binomial distributions [42]. The proportion of productive breeding sites will be compared between intervention and control hotspots by GEE models, adjusting for correlations between observations from the same clusters.

\section{Ethics considerations Ethics approval}

The study proposal received ethics approval from the Scientific Steering Committee (SSC), the ethical review committee (ERC) of the KEMRI Nairobi (proposal numbers SSC 2163, 2181 and 1589), the London School of Hygiene \& Tropical Medicine ethics committee (\#6111), and from Centers for Disease Control and Prevention (with exempt status).

\section{Informed consent}

Indoor residual spraying is to be conducted as part of the routine district-wide malaria control programme. Consent will be obtained orally at the compound by community health workers and spray operators recruited by MOPHS, as is consistent with their operating procedures. Ahead of targeted distribution of LLINs, informed, written consent will be sought at the house level from the head of the household or representative in the presence of an independent witness. Larviciding will be done after consulting with and receiving approval from the DOMC, the Kenyan Pest Control Product Board (PCPB), the district administrative, fisheries and health teams and after community meetings. Oral consent will be sought from owners of or persons responsible for any privately owned permanent breeding sites in the intervention areas (for example, fish ponds). Since most mosquito breeding sites are not restricted to particular households, consent at household level is not practical and approval from the community, DOMC and PCPB is considered adequate.

Before FSAT and cross-sectional surveys, informed written consent will be sought from all individuals and, if appropriate, their parents or guardians. If the signatory is not literate, a thumbprint will be obtained and confirmed by an independent witness. Assent forms will be signed by children between the ages of 13 and 17 years and by their parents or guardians. Each assent form will be accompanied by a consent form signed by the parent or guardian. All consent and assent forms will be countersigned by the staff member obtaining consent and a copy will be left at the household.

\section{Trial oversight}

Ethical and safety aspects of the study are overseen by an independent monitor. No data safety and monitoring board (DSMB) will be installed. Indoor residual spraying and LLINs form part of routine malaria control in Kenya and will be undertaken in collaboration with the DOMC and the district public health teams. Larviciding with Bti has been undertaken previously in neighbouring districts and has previously been shown to pose no health risk [43]. The proposed form of FSAT, where household members of parasite carriers are treated regardless of their parasite status by microscopy, is not part of the current malaria strategy of the Kenyan DOMC, although screening and treatment of asymptomatic parasite carriers is recommended [44]. Our FSAT approach is based on the assumption of a high proportion of submicroscopic infections among asymptomatic individuals [45], especially among household members of individuals with patent parasitaemia [46]. The drug used throughout the study, AL, is the first line antimalarial treatment in most of East Africa, including Kenya.

\section{Discussion}

Targeting interventions to hotspots of malaria transmission is frequently mentioned as a cost-effective approach for malaria control and elimination [2,4,5,47], although direct evidence for a community effect of hotspot-targeted interventions is currently unavailable. The present study aims to determine this effect in a cluster-randomized intervention trial.

Valuable information on how to quantify community effects of malaria control interventions comes from trials with ITNs [48]. Mortality rates [49], incidence of severe malaria [50], incidence of uncomplicated malaria [39,50], anaemia [39] and high-density parasitaemia [39] have been shown to be reduced in compounds without ITNs that were in close proximity of compounds with ITNs. Hawley and colleagues found that individuals living in control villages within $300 \mathrm{~m}$ of ITN villages in Kenya experienced a level of protection similar to that experienced by individuals living in ITN villages and that this was plausibly due to area-wide effects on vector densities and sporozoite-positive mosquitoes [39]. Despite similarities, hotspot-targeted interventions may differ considerably from untargeted ITN campaigns in their community impact. Mathematical simulation models suggest that the impact of hotspot-targeted interventions may be much larger than that of community-wide ITN distributions and may lead to local malaria elimination [4]. In line with this, our trial is powered to detect large effects on malaria transmission. However, two of the major assumptions underlying the optimistic model outcomes are incompletely understood. Firstly, the stability of hotspots is central to ensure sustainable community effects. Hotspots of (asymptomatic) parasite carriage are generally assumed to be stable $[4,10]$. However, a report that wind direction in relation to breeding site location may be a key element in determining the location of hotspots [12], suggests that local environmental factors may also influence the spatial stability of hotspots. We believe that our approach to 
define hotspots serologically may be less susceptible to (short-term) variations in wind direction or other ecological factors, since it effectively bases hotspot-detection on immunological markers of cumulative malaria exposure [27]. Secondly, a community effect of hotspot-targeted interventions strongly depends on mosquito mixing patterns. Mosquito mixing patterns are unlikely to be homogeneous. Reported site-fidelity where mosquitoes are likely to return to the same compounds $[51,52]$ remains to be confirmed but could considerably reduce the community effect of hotspot-targeted interventions. The most informative measure of mixing patterns may be an approach where parasite populations are tracked in human populations, inside and outside hotspots of malaria transmission.

Research on the impact of community interventions where 'herd coverage' is required to ensure effectiveness raises a number of practical issues. Similar to mass drug administration campaigns, high community coverage $[53,54]$ is required in our study to reduce $R_{0}$ to values below 1 . Our intervention is further challenged by a dependence on community participation in control measures that are only rolled out in a selected proportion of this community. Gaining community trust is essential to the study's success and we expect good participation rates after our lengthy sensitization process and strong involvement of community leaders and local workers in all aspects of the study preparation, intervention and evaluation.

Even with excellent participation rates, the nature of our intervention will remain susceptible to contamination from neighbouring hotspots. An ideal study setting would comprise a large number of geographically isolated clusters, each being an independent focus of malaria transmission, with clearly defined hotspots within these clusters [4]. Our real-life setting falls short of this ideal scenario. The continuous inhabitation in the area makes it unlikely that clusters are geographically isolated. We aim to minimize contamination from non-targeted malaria hotspots by incorporating an exclusion zone in our selection of eligible hotspots and in the selection of compounds in the evaluation phase. We nevertheless expect that there will be residual contamination that will be reflected in a spatial component in the effect of hotspot-targeted interventions: we expect the level of contamination to be highest in areas furthest away from the targeted hotspot and nearest to untargeted hotspots. Similarly, the effect of the intervention within the targeted hotspots may be largest in those compounds that are most remote from the nearest untargeted compound. Mathematical simulation models that incorporate heterogeneous malaria exposure [16,23,38] are expected to be valuable as an integral part of the evaluation of our intervention to assess the plausibility that a change in transmission intensity can be attributed to the intervention.
The current study is not designed to determine the effect of individual interventions. While simulations suggest that targeted interventions with LLINs and IRS will be sufficient to eliminate malaria locally [4], we chose a relatively comprehensive package of malaria control measures incorporating a wide variety of available interventions, targeting both the mosquito vector and the malaria parasite in humans. If findings from the current study prove promising, a next step will be to determine the optimum package of tools for hotspot-targeted interventions across a range of settings. This package will differ between different settings. Larviciding, for example, will be most beneficial in settings where breeding sites are discrete and well-defined [55-57] while the effects of IRS and ITNs will be affected by insecticide resistance, amongst other factors [48]. Importantly, follow-up studies should determine the cost-effectiveness of the hotspot approach to assess whether savings in the number of compounds that need to be targeted for conventional vector control in the absence of hotspot treatment outweigh the costs for hotspot-detection and coordination of hotspot interventions.

\section{Trial status}

The trial was actively recruiting participants at the time that the protocol was submitted for publication.

\section{Abbreviations}

ACT: artemisinin combination therapy; $\mathrm{AL}$ : artemether-lumefantrine; AMA1: apical membrane antigen; Bti: Bacillus thuringiensis israelensis; CDC: Centers for Disease Control; DOMC: (Kenyan) Division of Malaria Control; DSMB: Data Safety and Monitoring Board; EIR: entomologic inoculation rate;

ELISA: enzyme-linked immunosorbent assay; ERC: ethical review committee; FSAT: focal screen and treat; GEE: generalized estimating equations; GPS: global positioning system; Hb: haemoglobin; HRP-2: histidine rich protein-2; ICON: lambda cyhalothrin (ICON 10 CS); ID: identification; IgG: immunoglobulin G; IRS: indoor residual spraying; ITN: insecticide-treated net; KEMRI: Kenya Medical Research Institute; LLIN: long-lasting insecticidetreated net; MOPHS: Ministry of Public Health and Sanitation; MSP-

1: merozoite surface protein 1; OD: optical density; PCPB: Kenyan Pest Control Product Board; PCR: polymerase chain reaction; PDA: personal digital assistant; pLDH: plasmodium lactate dehydrogenase; PSC: pyrethrum spray catch; $R_{0}$ : basic reproduction number; RDT: rapid diagnostic test;

RTI: Research Triangle Institute; SCR: sero-conversion rate; SSC: Scientific Steering Committee.

\section{Competing interests}

The authors declare that they have no competing interests.

\section{Authors' contributions}

Trial design: TB, JS, IK, RS, CD, JC; design of intervention packages JS, AB, GS, $J V, N B, K L, M D, J C$; design of analytical plan: TB, JTG, IK, KL, MD, RS, CD, JC; preparation and conduct of surveys: JS, $A B, G S$; contribution of reagents EJR, contributed to manuscript preparation TB, JS, AB, GS, JTG, IK, EJR, JV, NB, MD, $R S, C D, J C$. All authors read and approved the final manuscript.

\section{Acknowledgements}

We thank project staff, the community of Kabondo and Kasipul, Rachuonyo South, and KEMRI/CDC Kisumu. We also thank the project scientific advisory board consisting of Sodiomon Sirima (Centre National de Recherche et de Formation sur le Paludisme, Ouagadougou, Burkina Faso), Umberto d'Alessandro (Medical Research Council Laboratories, Fajara, The Gambia) and Philip Bejon (Kenya Medical Research Institute, Wellcome Trust Research 
Programme, Kilifi, Kenya). This project is funded by the Bill and Melinda Gates Foundation, under the Malaria Transmission Consortium, Grant No.45114 and the Grand Challenge Grant No. OPP1024438. This manuscript has been approved by the Director of the Kenya Medical Research Institute.

\section{Author details}

'Department of Immunology \& Infection; Faculty of Infectious and Tropical Diseases, London School of Hygiene and Tropical Medicine, London, UK.

${ }^{2}$ Radboud University Nijmegen Medical Centre, Nijmegen, the Netherlands. ${ }^{3}$ Department of Disease Control; Faculty of Infectious and Tropical Diseases, London School of Hygiene and Tropical Medicine, London, UK. ${ }^{4}$ MRC Centre for Outbreak Analysis \& Modelling, Department of Infectious Disease Epidemiology, Imperial College London, London, UK. ${ }^{5} \mathrm{MRC}$ Tropical Epidemiology Group, Department of Infectious Disease Epidemiology, London School of Hygiene and Tropical Medicine, London, UK. ${ }^{6}$ Department of Parasitology, Biomedical Primate Research Centre, Rijswijk, The Netherlands. ${ }^{1}$ Kenya Medical Research Institute, Centre for Global Health Research, Kisumu, Kenya. ${ }^{8}$ Centers for Disease Control and Prevention, Division of Parasitic Diseases and Malaria, Atlanta, GA, USA.

Received: 17 August 2012 Accepted: 16 January 2013

Published: 2 February 2013

\section{References}

1. Manning SD, Woolhouse ME, Ndamba J: Geographic compatibility of the freshwater snail Bulinus globosus and schistosomes from the Zimbabwe highveld. Int J Parasitol 1995, 25:37-42.

2. Woolhouse ME, Dye C, Etard JF, Smith T, Charlwood JD, Garnett GP, Hagan P, Hii JL, Ndhlovu PD, Quinnell RJ, Watts CH, Chandinawa SK, Anderson RM: Heterogeneities in the transmission of infectious agents: implications for the design of control programs. Proc Natl Acad Sci USA 1997, 94:338-342.

3. Criscione CD, Anderson JD, Sudimack D, Subedi J, Upadhayay RP, Jha B, Williams $K D$, Williams-Blangero $S$, Anderson TJ: Landscape genetics reveals focal transmission of a human macroparasite. PLoS Negl Trop Dis 2010, 4:e665.

4. Bousema T, Griffin JT, Sauerwein RW, Smith DL, Churcher TS, Takken W, Ghani A, Drakeley C, Gosling R: Hitting hotspots: spatial targeting of malaria for control and elimination. PLoS Med 2012, 9:e1001165.

5. Carter R, Mendis KN, Roberts D: Spatial targeting of interventions against malaria. Bull World Health Organ 2000, 78:1401-1411.

6. Oesterholt MJ, Bousema JT, Mwerinde OK, Harris C, Lushino P, Masokoto A, Mwerinde H, Mosha FW, Drakeley CJ: Spatial and temporal variation in malaria transmission in a low endemicity area in northern Tanzania. Malar J 2006, 5:98.

7. Clark TD, Greenhouse B, Njama-Meya D, Nzarubara B, Maiteki-Sebuguzi C, Staedke SG, Seto E, Kamya MR, Rosenthal PJ, Dorsey G: Factors determining the heterogeneity of malaria incidence in children in Kampala. Uganda. J Infect Dis 2008, 198:393-400.

8. Kreuels B, Kobbe R, Adjei S, Kreuzberg C, von Reden C, Bater K, Klug S, Busch W, Adjei O, May J: Spatial variation of malaria incidence in young children from a geographically homogeneous area with high endemicity. J Infect Dis 2008, 197:85-93.

9. Bousema T, Drakeley C, Gesase S, Hashim R, Magesa S, Mosha F, Otieno S, Carneiro I, Cox J, Msuya E, Kleinschmidt I, Maxwell C, Greenwood B, Riley E, Sauerwein R, Chandramohan D, Gosling R: Identification of hot spots of malaria transmission for targeted malaria control. J Infect Dis 2010, 201:1764-1774.

10. Bejon P, Williams TN, Liljander A, Noor AM, Wambua J, Ogada E, Olotu A, Osier FH, Hay SI, Farnert A, Marsh K: Stable and unstable malaria hotspots in longitudinal cohort studies in Kenya. PLoS Med 2010, 7:e1000304.

11. Ghebreyesus TA, Haile M, Witten KH, Getachew A, Yohannes AM, Yohannes M, Teklehaimanot HD, Lindsay SW, Byass P: Incidence of malaria among children living near dams in northern Ethiopia: community based incidence survey. Br Med J 1999, 319:663-666.

12. Midega JT, Smith DL, Olotu A, Mwangangi JM, Nzovu JG, Wambua J, Nyangweso G, Mbogo CM, Christophides GK, Marsh K, Bejon P: Wind direction and proximity to larval sites determines malaria risk in Kilifi District in Kenya. Nat Commun 2012, 3:674.

13. Ghebreyesus TA, Haile M, Witten KH, Getachew A, Yohannes M, Lindsay SW, Byass $P$ : Household risk factors for malaria among children in the Ethiopian highlands. Trans R Soc Trop Med Hyg 2000, 94:17-21.
14. Gamage-Mendis AC, Carter R, Mendis C, De Zoysa AP, Herath PR, Mendis $\mathrm{KN}$ : Clustering of malaria infections within an endemic population: risk of malaria associated with the type of housing construction. Am J Trop Med Hyg 1991, 45:77-85.

15. Mackinnon MJ, Mwangi TW, Snow RW, Marsh K, Williams TN: Heritability of malaria in Africa. PLoS Med 2005, 2:e340.

16. Smith DL, McKenzie FE, Snow RW, Hay SI: Revisiting the basic reproductive number for malaria and its implications for malaria control. PLOS Biol 2007, 5:e42.

17. Bousema T, Kreuels B, Gosling R: Adjusting for heterogeneity of malaria transmission in longitudinal studies. J Infect Dis 2011, 204:1-3.

18. Clarke SE, Bogh C, Brown RC, Walraven GE, Thomas CJ, Lindsay SW: Risk of malaria attacks in Gambian children is greater away from malaria vector breeding sites. Trans R Soc Trop Med Hyg 2002, 96:499-506.

19. Cook J, Kleinschmidt I, Schwabe C, Nseng G, Corran PH, Bousema T, Riley EM, Drakeley CJ: Serological markers identify heterogeneity of effectiveness of malaria control interventions on Bioko Island, equatorial Guinea. PLoS One 2011, 6:e25137.

20. Bejon P, Turner L, Lavstsen T, Cham G, Olotu A, Drakeley CJ, Lievens M Vekemans J, Savarese B, Lusingu J, von Seidlein L, Bull PC, Marsh K, Theander TG: Serological evidence of discrete spatial clusters of Plasmodium falciparum parasites. PLoS One 2011, 6:e21711.

21. Bousema T, Youssef RM, Cook J, Cox J, Alegana VA, Amran J, Noor AM Snow RW, Drakeley C: Serologic markers for detecting malaria in areas of low endemicity, Somalia, 2008. Emerg Infect Dis 2010, 16:392-399.

22. Stone W, Bousema T, Jones S, Gesase S, Hashim R, Gosling R, Carneiro I, Chandramohan D, Theander T, Ronca R, Modiano D, Arcà B, Drakeley C: IgG responses to Anopheles gambiae salivary antigen gSG6 detect variation in exposure to malaria vectors and disease risk. PLoS One 2012, 7:e40170

23. Stuckey EM, Stevenson JC, Cooke MK, Owaga C, Marube E, Oando G, Hardy D, Drakeley C, Smith TA, Cox J, Chitnis N: Simulation of malaria epidemiology and control in the highlands of Western Kenya. Malar J 2012, 11:357.

24. Plowe CV, Djimde A, Bouare M, Doumbo O, Wellems TE: Pyrimethamine and proguanil resistance-conferring mutations in Plasmodium falciparum dihydrofolate reductase: polymerase chain reaction methods for surveillance in Africa. Am J Trop Med Hyg 1995, 52:565-568.

25. Steenkeste $N$, Incardona S, Chy S, Duval L, Ekala MT, Lim P, Hewitt S, Sochantha T, Socheat D, Rogier C, Mercereau-Puijalon O, Fandeur T, Ariey F: Towards high-throughput molecular detection of Plasmodium: new approaches and molecular markers. Malar J 2009, 8:86.

26. Hsiang MS, Lin M, Dokomajilar C, Kemere J, Pilcher CD, Dorsey G, Greenhouse B: PCR-based pooling of dried blood spots for detection of malaria parasites: optimization and application to a cohort of Ugandan children. J Clin Microbiol 2010, 48:3539-3543.

27. Drakeley CJ, Corran PH, Coleman PG, Tongren JE, McDonald SL, Carneiro I, Malima R, Lusingu J, Manjurano A, Nkya WM, Lemnge MM, Cox J, Reyburn $\mathrm{H}$, Riley EM: Estimating medium- and long-term trends in malaria transmission by using serological markers of malaria exposure. Proc Nat Acad Sci USA 2005, 102:5108-5113.

28. Corran PH, Cook J, Lynch C, Leendertse H, Manjurano A, Griffin J, Cox J, Abeku T, Bousema T, Ghani AC, Drakeley C, Riley E: Dried blood spots as a source of anti-malarial antibodies for epidemiological studies. Malar J 2008, 7:195.

29. Wilson S, Booth M, Jones FM, Mwatha JK, Kimani G, Kariuki HC, Vennervald BJ, Ouma JH, Muchiri E, Dunne DW: Age-adjusted Plasmodium falciparum antibody levels in school-aged children are a stable marker of microgeographical variations in exposure to Plasmodium infection. BMC Infect Dis 2007, 7:67.

30. SaTScan ${ }^{\mathrm{TM}}$ Software for the spatial, temporal, and space-time scan statistics. http://www.satscan.org/.

31. Kulldorff M, Huang L, Pickle L, Duczmal L: An elliptic spatial scan statistic. Stat Med 2006, 25:3929-3943.

32. Fillinger U, Kannady K, William G, Vanek MJ, Dongus S, Nyika D, Geissbühler Y, Chaki PP, Govella NJ, Mathenge EM, Singer BH, Mshinda H, Lindsay SW, Tanner M, Mtasiwa D, de Castro MC, Killeen GF: A tool box for operational mosquito larval control: preliminary results and early lessons from the Urban Malaria Control Programme in Dar es Salaam. Tanzania. Malar J 2008, 7:20.

33. World Health Organization: Manual on Practical Entomology in Malaria, Part II. Geneva; 1975

34. Mboera LE, Kihonda J, Braks MA, Knols BG: Influence of Centers for Disease Control light trap position, relative to a human-baited bed net, on 
catches of Anopheles gambiae and Culex quinquefasciatus in Tanzania. Am J Trop Med Hyg 1998, 59:595-596.

35. Govella NJ, Chaki PP, Mpangile JM, Killeen GF: Monitoring mosquitoes in urban Dar es Salaam: evaluation of resting boxes, window exit traps, CDC light traps. Ifakara tent traps and human landing catches. Parasit Vectors 2011, 4:40

36. Odetoyinbo JA: Preliminary investigation on the use of a light-trap for sampling malaria vectors in the Gambia. Bull World Health Organ 1969, 40:547-560

37. Wirtz RA, Burkot TR, Graves PM, Andre RG: Field evaluation of enzymelinked immunosorbent assays for Plasmodium falciparum and Plasmodium vivax sporozoites in mosquitoes (Diptera: Culicidae) from Papua New Guinea. J Med Entomol 1987, 24:433-437.

38. Griffin JT, Hollingsworth TD, Okell LC, Churcher TS, White M, Hinsley W, Bousema T, Drakeley CJ, Ferguson NM, Basanez M-G, Ghani AC: Strategies towards Plasmodium falciparum malaria elimination in Africa using currently available tools. PLoS Med 2010, 6:e20179.

39. Hawley WA, Phillips-Howard PA, ter Kuile FO, Terlouw DJ, Vulule JM, Ombok M, Nahlen BL, Gimnig JE, Kariuki SK, Kolczak MS, Hightower AW: Community-wide effects of permethrin-treated bed nets on child mortality and malaria morbidity in western Kenya. Am J Trop Med Hyg 2003, 68:121-127.

40. Hayes RJ, Bennett S: Simple sample size calculation for clusterrandomized trials. Int J Epidemiol 1999, 28:319-326.

41. Goldstein H: Multilevel Statistical Models. 4th edition. London: John Wiley and Sons Ltd; 2010.

42. Crespi CM, Wong WK, Mishra SI: Using second-order generalized estimating equations to model heterogeneous intraclass correlation in cluster-randomized trials. Stat Med 2009, 28:814-827.

43. World Health Organization: Report of the Seventh WHOPES Working Group Meeting. Review of VectoBac WG, Permanet, Gokilaht-S 5EC. Geneva: WHO/ CDS/WHOPES/2004.8; 2004

44. Division of Malaria Control: National Malaria Strategy 2009-2017: Towards a Malaria-free Kenya. Nairobi: Division of Malaria Control; Ministry of Public Health and Sanitation; 2009.

45. Okell LC, Ghani AC, Lyons E, Drakeley CJ: Submicroscopic infection in Plasmodium falciparum-endemic populations: a systematic review and meta-analysis. J Infect Dis 2009, 200:1509-1517.

46. Stresman GH, Kamanga A, Moono P, Hamapumbu H, Mharakurwa S, Kobayashi T, Moss WJ, Shiff C: A method of active case detection to target reservoirs of asymptomatic malaria and gametocyte carriers in a rural area in Southern Province. Zambia. MalarJ 2010, 9:265.

47. Carter R: Spatial simulation of malaria transmission and its control by malaria transmission blocking vaccination. Int J Parasitol 2002, 32:1617-1624.

48. Killeen GF, Okumu FO, N'Guessan R, Coosemans M, Adeogun A, Awolola S, Etang J, Dabire RK, Corbel V: The importance of considering communitylevel effects when selecting insecticidal malaria vector products. Parasit Vectors 2011, 4:160.

49. Binka FN, Indome F, Smith T: Impact of spatial distribution of permethrinimpregnated bed nets on child mortality in rural northern Ghana. Am J Trop Med Hyg 1998, 59:80-85.

50. Howard SC, Omumbo J, Nevill C, Some ES, Donnelly CA, Snow RW: Evidence for a mass community effect of insecticide-treated bednets on the incidence of malaria on the Kenyan coast. Trans $R$ Soc Trop Med Hyg 2000, 94:357-360.

51. McCall PJ, Mosha FW, Njunwa KJ, Sherlock K: Evidence for memorized sitefidelity in Anopheles arabiensis. Trans R Soc Trop Med Hyg 2001, 95:587-590.

52. Charlwood JD, Graves PM, Marshall TF: Evidence for a 'memorized' home range in Anopheles farauti females from Papua New Guinea. Med Vet Entomol 1988, 2:101-108.

53. Shekalaghe SA, Drakeley C, van den Bosch S, ter Braak R, van den Bijllaardt W, Mwanziva C, Semvua S, Masokoto A, Mosha F, Teelen K, Hermsen R, Okell L, Gosling R, Sauerwein R, Bousema T: A cluster-randomized trial of mass drug administration with a gametocytocidal drug combination to interrupt malaria transmission in a low endemic area in Tanzania. Malar $\lrcorner$ 2011, 10:247.

54. Okell LC, Griffin J, Kleinschmidt I, Hollingsworth TD, Churcher T, White M, Bousema T, Drakeley CJ, Ghani A: The potential contribution of mass treatment to the control of Plasmodium falciparum malaria. PLoS One 2011, 6:e20179.
55. Fillinger U, Ndenga B, Githeko A, Lindsay SW: Integrated malaria vector control with microbial larvicides and insecticide-treated nets in western Kenya: a controlled trial. Bull World Health Organ 2009, 87:655-665.

56. Fillinger U, Sonye G, Killeen GF, Knols BG, Becker N: The practical importance of permanent and semipermanent habitats for controlling aquatic stages of Anopheles gambiae sensu lato mosquitoes: operational observations from a rural town in western Kenya. Trop Med Int Health 2004, 9:1274-1289.

57. Geissbühler Y, Kannady K, Chaki PP, Emidi B, Govella NJ, Mayagaya V, Kiama M, Mtasiwa D, Mshinda H, Lindsay SW, Tanner M, Fillinger U, de Castro MC, Killeen GF: Microbial larvicide application by a large-scale, communitybased program reduces malaria infection prevalence in urban Dar es Salaam. Tanzania. PLoS One 2009, 4:e5107.

doi:10.1186/1745-6215-14-36

Cite this article as: Bousema et al:: The impact of hotspot-targeted interventions on malaria transmission: study protocol for a clusterrandomized controlled trial. Trials 2013 14:36.

\section{Submit your next manuscript to BioMed Central and take full advantage of:}

- Convenient online submission

- Thorough peer review

- No space constraints or color figure charges

- Immediate publication on acceptance

- Inclusion in PubMed, CAS, Scopus and Google Scholar

- Research which is freely available for redistribution 